\title{
Temperature dependence of underdense nanostructure formation in tungsten under helium irradiation
}

\section{Valles, G.}

2017-07

Valles , G, Martin-Bragado , I , Nordlund , K , Lasa , A , Björkas , C , Safi , E , Perlado , J M \& Rivera , A 2017 , ' Temperature dependence of underdense nanostructure formation in tungsten under helium irradiation ' , Journal of Nuclear Materials , vol. 490 , pp. 108-114 . https://doi.org/10.1016/j.jn

http://hdl.handle.net/10138/308765

https://doi.org/10.1016/j.jnucmat.2017.04.021

cc_by_nc_nd

acceptedVersion

Downloaded from Helda, University of Helsinki institutional repository.

This is an electronic reprint of the original article.

This reprint may differ from the original in pagination and typographic detail.

Please cite the original version. 


\title{
Temperature dependence of underdense
}

\section{nanostructure formation in tungsten under helium irradiation}

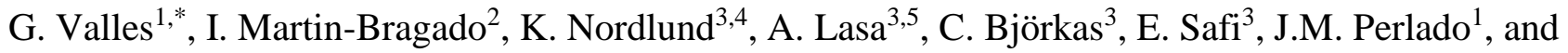 \\ A. Rivera ${ }^{1}$ \\ ${ }^{1}$ Instituto de Fusión Nuclear, Universidad Politécnica de Madrid, C/ José Gutiérrez Abascal 2, 28006, \\ Madrid, Spain \\ ${ }^{2}$ UCAM, Universidad Católica de Murcia, Campus de los Jerónimos, Guadalupe, 30107 Murcia, Spain \\ ${ }^{3}$ Department of Physics, University of Helsinki. P.O. Box 43, Helsinki FI-00014, Finland \\ ${ }^{4}$ National Research Nuclear University MEPhI, 115409 Moscow, Russia \\ ${ }^{5}$ Oak Ridge National Laboratory, Oak Ridge, TN37831-6169, USA \\ *e-mail address: gonzalovallesalberdi@ hotmail.com
}

\begin{abstract}
Recently, tungsten has been found to form a highly underdense nanostructured morphology ("W fuzz") when bombarded by an intense flux of He ions, but only in the temperature window 900-2000 K. Using object kinetic Monte Carlo simulations (pseudo-3D simulations) parameterized from first principles, we show that this temperature dependence can be understood based on He and point defect clustering, cluster growth, and detrapping reactions. At low temperatures ( $<900 \mathrm{~K})$, fuzz does not grow because almost all He is trapped in very small He-vacancy clusters. At high temperatures (>2300 K), all He is detrapped from clusters, preventing the formation of the large clusters that lead to fuzz growth in the intermediate temperature range.
\end{abstract}

Keywords: Tungsten, helium irradiation, fuzz nanostructures, OKMC simulations, temperature

\section{Highlights}

- OKMC simulation of temperature window for fuzz formation

- Stable He-V clusters prevent fuzz formation at low temperatures

- Dissociation of He-V clusters prevent fuzz formation at high temperatures

- Fuzz formation rate increases with increasing temperature

- An incubation fluence observed in the simulation, similar to experimental observations 


\section{Introduction}

Tungsten is the prime plasma facing material (PFM) candidate for the divertor of future fusion devices due to its properties, such as low sputtering yield, low tritium retention, high melting point and high thermal conductivity [1-3]. Sections of the divertor closest to the strike point will reach high temperatures $(\geq 1000 \mathrm{~K})$ while exposed to large particle fluxes $\left(>10^{23} \mathrm{~m}^{-2} \mathrm{~s}^{-1}\right)$ of low-energy hydrogen and helium ions [4,5]. Experimental studies of $\mathrm{W}$ exposed to these conditions report on the formation of voids and underdense W nanostructures (so called "fuzz"), shown in Figure 1. Fuzz is formed not only due to (low energy) He irradiation of metals such as tungsten, molybdenum [6] or palladium [7], but also when exposed to mixed $\mathrm{D}_{2}$-He plasmas [8]. Fuzz formation could be beneficial offering novel properties for these metals, e.g. as a means to make an underdense nanoporous surface layer with high chemical reactivity for catalytic activity. However, in the context of nuclear fusion, the formation of these nanostructures seems to over all be detrimental [9]. Several properties of W as PFM have been reported to worsen with the appearance of fuzz (voids and W nanostructures), such as decrease of the thermal conductivity and optical reflectivity of $\mathrm{W}$ [10-12] and surface effects under transient heat loads that might lead to an increase in the $\mathrm{W}$ release [13].

Experiments of He irradiation in linear divertor-plasma simulators [4] and with magnetron sputtering devices [14] provided similar results regarding fuzz formation. Baldwin et al. [4] studied the kinetics of fuzz growth, finding that it scales with the square root of time, $t^{1 / 2}$, at the studied temperatures: 1120 and $1320 \mathrm{~K}$. At $1120 \mathrm{~K}$ the He energy threshold for nanostructure formation was determined to be around $35 \mathrm{eV}$ [15] and the growth rate to increase with flux [8]. The role of defects in these processes has also been reported in Ref. [16]. A clear influence of temperature has been observed, revealing a limited temperature window for fuzz growth between, approximately, 900-2000 K [17-19]. Some theoretical studies have been carried out in order to explain the growth rate dependence with $t^{1 / 2}$. Modeling performed in Refs. [20,21] showed that the growth rate is driven by the balance of two processes: formation of He bubbles and their rupture at the surface. Along these lines, a four-step process has been proposed by Ito et al. [22,23] at $2000 \mathrm{~K}$ : (i) He ions penetrate the W surface; (ii) He diffusion and agglomeration even at interstitial sites occurs; (iii) He bubbles grow at both vacancy and the interstices and (iv) based on hybrid MD-MC simulations, they explained how He bubbles burst, forming fuzzy nanostructures. Further, the dynamics of small He clusters formed near the $\mathrm{W}$ surface has been extensively studied [24-29]. Nevertheless, no quantitative studies were found in the literature addressing why fuzz formation occurs only in this limited temperature window.

In the present study we report on an Object Kinetic Monte Carlo (OKMC) simulation of low energy He irradiation $(60 \mathrm{eV})$ of W. Simulating a broad range of temperatures, from 700 to $2500 \mathrm{~K}$, we have determined a fuzz growth temperature window, in agreement with published experiments [17-19]. In summary, at low temperatures $(700 \mathrm{~K})$, the great majority of He atoms are retained in monovacancies 
$\left(\mathrm{He}_{n} \mathrm{~V}_{1}\right.$ clusters), which remain stable. At intermediate temperatures (900-1900 K), He atoms are retained inside larger $\mathrm{He}_{n} \mathrm{~V}_{m}$ clusters, which grow to trigger the formation of fuzz-like structures. At high temperatures $(2500 \mathrm{~K}), \mathrm{He}$ atoms and vacancies are emitted from small $\mathrm{He}_{n} \mathrm{~V}_{m}$ clusters $(n<40, m<10)$, preventing the formation of larger $\mathrm{He}_{n} \mathrm{~V}_{m}$ clusters and the growth of fuzz nanostructures.

\section{Simulation methods}

Our simulations have been performed using the open-source code MMonCa [30], parameterized with in house Density Functional Theory (DFT) calculations (see Table 3 of Ref. [31]), as well as values from literature [32,33], with the exception that pure He clusters $\left(\mathrm{He}_{n}\right)$ are assumed immobile and cannot emit He atoms. Although small clusters can migrate [34,35], the assumption is justified by cluster diffusion being slowed down with the cluster size and strongly suppressed by impurities and interstices $[22,23,36]$. In addition, trap mutation reactions [37,38] have been considered. These trap mutation reactions, enhanced near the irradiation surface [39-41], constitute an efficient way of He trapping $\left(\mathrm{He}_{n} \rightarrow \mathrm{He}_{n} \mathrm{~V}+\mathrm{I}\right)$. The maximum He/V ratio allowed in these simulations is 9, i.e., $\mathrm{He}_{9 m} \mathrm{~V}_{m}$ clusters. This limit is based on theoretical calculations $[42,43]$ as well as on experimental observations [44]. As DFT calculations are only possible for small clusters, we have approximated the DFT values to a power law for large $\mathrm{He}_{n} \mathrm{~V}_{m}$ clusters [31]:

$$
E_{b}\left(H e \rightarrow H e_{n-1} V_{m}\right)=4.43 \cdot\left(\frac{n}{m}\right)^{-0.28} \text {, }
$$

where $E_{b}$ is the binding energy and $n$ and $m$, the number of He atoms and vacancies in the cluster, respectively. A maximum value for the binding energy of a He atom to a $\mathrm{He}_{n} \mathrm{~V}_{m}$ cluster was set to $\sim 6 \mathrm{eV}[45]$.

Thin $\mathrm{W}$ boxes of $40(X) \times 4(Y) \times 20(Z$, height $) \mathrm{nm}^{3}$ were used to carry out pseudo-3D simulations. The width of the simulation box ( $X$ dimension) was taken as large as the expected size of the tendrils that will be formed $(30 \pm 10 \mathrm{~nm}$, as reported experimentally in Ref. [46]). The depth of the box (20 nm) allows simulating the migration of He ions (for an implantation depth of $\sim 1.3 \mathrm{~nm}$ as calculated by SRIM, see below). Full 3D boxes were very computationally demanding, turning out impractical. Periodic boundary conditions (PBCs) were used only in the two largest lateral surfaces (normal to $Y$ direction). Free surfaces were considered in the two smaller surfaces (normal to $X$ direction), in order to let the material expand along $X$ and $Z$ directions and thus, reproduce the evolution of the surface roughness (Figure 2). The top surface (normal to the $Z$-axis) was considered as a desorption surface, whereas atoms reaching the bottom surface were considered to move further deep into the bulk and thus ignored. Each simulation box was composed of small cells $\left(0.5 \times 0.5 \times 0.5 \mathrm{~nm}^{3}\right)$, named mesh elements. The mesh elements out of the $\mathrm{W}$ box were considered as vacuum. If the volume of the He atoms retained in a given cluster (assuming $0.0033 \mathrm{~nm}^{3}$ per single $\mathrm{He}$ ) was equal or larger than the volume of a mesh element $\left(0.125 \mathrm{~nm}^{3}\right)$, then the closest vacuum mesh element above the surface was changed to a $\mathrm{W}$ mesh 
element, thus simulating the surface growth. When the distance of a cluster to the desorption surface was lower than $0.2 \mathrm{~nm}$, the whole cluster was considered to burst. Helium atoms were discarded from the simulation and a number of $\mathrm{W}$ fuzz mesh elements equal to the volume of released He atoms-change was changed to vacuum, thus simulating the bubble burst. This approach to fuzz growth is similar to that observed in MD simulations presented in Ref. [21]. However, with the current methodology, fuzz grows not only normal to the surface $(Z)$ but also along the $X$ direction, not included in the former study. The implantation depth of He ions was calculated with the SRIM code [47], by irradiating W with He ions at $60 \mathrm{eV}$. Helium ions were implanted following a Gaussian distribution in depth taking into account the surface height where and when implanted (see Figure 3).

The flux was $5 \times 10^{22} \mathrm{~m}^{-2} \mathrm{~s}^{-1}$, taking into account the reflection yield provided by SRIM. When a He ion is reflected, it was not implanted in the simulation box but time was allowed to evolve. Fuzz was considered to be the $\mathrm{W}$ region that grows above the initial surface layer (Figure 2). Helium irradiation times of up to $3 \mathrm{~s}$, at impacting energies of $60 \mathrm{eV}$, were simulated at eight different temperatures: low temperature $(700 \mathrm{~K})$, intermediate temperatures $(900,1120,1320,1500,1700,1900,2100$ and $2300 \mathrm{~K})$ and high temperature $(2500 \mathrm{~K})$. In the case of $1700 \mathrm{~K}$ only results up to $1.25 \mathrm{~s}$ were obtained. The high temperature cases $(1900,2100$ and $2300 \mathrm{~K}$ ) were only simulated up to $0.25 \mathrm{~s}$. At these temperatures, the high number of thermally activated reactions leads to very high computational times. Nevertheless, the temperatures reported in this study constitute a representative set to study the temperature influence on fuzz formation.

\section{Results and discussion}

The modelled W fuzz growth after $3 \mathrm{~s}$ of He irradiation is shown in Figure 2. At low temperature $(700 \mathrm{~K})$, the whole simulation box (20 nm deep) becomes populated by $\mathrm{He}_{n} \mathrm{~V}_{m}$ clusters. However, these clusters are too small to cause any significant surface-, and hence, fuzz-growth. At intermediate temperatures, fuzz growth due to the accumulation of He atoms in larger $\mathrm{He}_{n} \mathrm{~V}_{m}$ clusters is observed: at $900 \mathrm{~K}$, fuzz slightly grows, only normal to the surface. Between 1120 and $1500 \mathrm{~K}$, there is a significant surface growth in both directions, normal to the surface $(Z)$ and along the $X$ axis. The higher the temperature, the more pronounced the fuzz growth. This trend is also observed at 1700 and $1900 \mathrm{~K}$ (see Figure 4). At $2100 \mathrm{~K}$ starts a change in the regime of fuzz growth and He retention (see below) that ends at high temperature $(2500 \mathrm{~K})$. Finally, at $2500 \mathrm{~K}$, neither fuzz growth nor He retention takes place. These results are in very good agreement with the experimentally reported temperature window for fuzz growth (900-2000 K) [17-19]. It is noteworthy that our modeling uses a general parameterization for He irradiation in $\mathrm{W}$, which was previously employed to reproduce very different irradiation conditions $[31,48,49]$. Moreover, in our simulations the mean diameter of $\mathrm{He}_{n} \mathrm{~V}_{m}$ clusters at a fluence of $1.5 \times 10^{23} \mathrm{~m}^{-2}$ is 0.36 and $0.44 \mathrm{~nm}$ at 700 and $900 \mathrm{~K}$, respectively. These results are in very good agreement with experimental values of He nano-bubbles mean diameter of $0.36\left(1.2 \times 10^{23} \mathrm{~m}^{-2}\right.$ at 623 - 
$673 \mathrm{~K})$ and $0.62 \mathrm{~nm}\left(0.62 \mathrm{~nm}\right.$, at $10^{23} \mathrm{~m}^{-2}$ and $\left.1073 \mathrm{~K}\right)$, as reported in Refs. [50,51]. Nevertheless, the ellipsoidal shape reported in the experiments cannot be reproduced with our code, which considers the $\mathrm{He}_{n} \mathrm{~V}_{m}$ clusters as spheres.

The fuzz height for different temperatures as a function of time (i.e., fluence) is presented in Figure 4. Over all, the growth rate increases with temperature. At low temperature (700 K), the absence of fuzz growth previously described is observed. At $900 \mathrm{~K}$ a slightly fuzz growth is seen, which is speed up between 1120 and $1500 \mathrm{~K}$ (up to 14.5, 56 and $94 \mathrm{~nm}$, respectively, at the end of the $3 \mathrm{~s}$ simulation). Fuzz growth is much faster at 1700, 1900 and $2100 \mathrm{~K}$, as observed even for short irradiation times. At $2300 \mathrm{~K}$, the fuzz growth rate decreases (with respect to 1900 and $2100 \mathrm{~K}$ ). It slows down as He emission from $\mathrm{He}_{n} \mathrm{~V}_{m}$ clusters increases due to the high temperature, making more difficult the growth of large $\mathrm{He}_{n} \mathrm{~V}_{m}$ clusters. This balance between He emission and cluster growth is discussed in detail later in this Section. At high temperature $(2500 \mathrm{~K})$, no fuzz growth occurs at all. The fuzz growth obtained from experimental data at 1120 and $1320 \mathrm{~K}$ (square root temporal dependence) [4] is also plotted. For short times scales, the temporal evolution obtained in our simulations does not follow the square root dependence, see Figure 4(b). A recent report [9] indicates that fuzz growth following the square root dependence only starts after accumulating an incubation fluence. Although the experiments were carried out at different irradiation conditions (He ion energy, flux and temperature), the incubation fluence is qualitatively comparable to our simulation results (see animation in the Supplementary Material). It can also be observed that the higher the temperature, the lower the incubation fluence, being almost negligible from $1700 \mathrm{~K}$. A full description of the temporal evolution is anyhow out of the scope of this study. Detailed experiments at low fluences for different temperatures and fluxes would allow for better understanding and prediction of the incubation fluence as a threshold for fuzz formation.

It is noteworthy that He retention alone (shown as a function of time and fluence for the temperatures considered in this work in Figure 5) cannot explain fuzz growth. In fact, up to irradiation times of $2.5 \mathrm{~s}$, the He retention fraction at $700 \mathrm{~K}$ (a temperature showing no fuzz growth) is higher than that at 900 , 1120,1320 and $1500 \mathrm{~K}$ (which show fuzz formation). Helium retention increases up to a temperature of $1900 \mathrm{~K}$. At $2100 \mathrm{~K}$ and higher temperatures, the He retention decreases as the He emission from clusters starts to play a more important role. An analysis of the He population in different types of clusters evidences that at low temperatures, the incoming He ions are trapped at first in pure $\mathrm{He}_{n}$ clusters. Once they reach 9 atoms $\left(\mathrm{He}_{9}\right)$ at $700 \mathrm{~K}$, they emit a self-interstitial atom (SIA) becoming stable $\mathrm{He}_{9} \mathrm{~V}_{1}$ clusters (trap mutation). Indeed, $95.9 \%$ of $\mathrm{He}$ is retained in $\mathrm{He}_{9} \mathrm{~V}_{1}$ clusters (see Figure 6), indicating almost no coalescence of clusters. The absence of large $\mathrm{He}_{n} \mathrm{~V}_{m}$ clusters suppresses fuzz formation.

At $900 \mathrm{~K}$, a similar process is observed regarding trap mutation: only $\mathrm{He}_{9}$ clusters are able to emit a SIA to become $\mathrm{He}_{9} \mathrm{~V}_{1}$. However, the crucial difference is that at this temperature, He atoms are emitted from $\mathrm{He}_{n} \mathrm{~V}_{m}$ clusters. On the one hand, this lowers He retention. On the other hand, some of the emitted 
He atoms are trapped in the vicinity of the emitting clusters, favoring coalescence of existing and new $\mathrm{He}_{n} \mathrm{~V}_{m}$ clusters, resulting in the formation of larger $\mathrm{He}_{n} \mathrm{~V}_{m}$ clusters that promote fuzz growth.

At higher temperatures $(1120,1320$ and $1500 \mathrm{~K})$, He retention decreases up to irradiation times of $1 \mathrm{~s}$. Beyond $1 \mathrm{~s}$, the presence of the already formed $\mathrm{He}_{n} \mathrm{~V}_{m}$ clusters increases the emission of He atoms, and thus the coalescence between $\mathrm{He}_{n} \mathrm{~V}_{m}$ clusters. As a result, He retention increases in a similar way for the three temperatures, resulting in a higher retention than at $900 \mathrm{~K}$. At these temperatures, fuzz growth occurs not only normal to the irradiation surface but also along the $X$ direction, allowing more volume for He trapping and thus increasing the retained He fraction. Further, the number of He atoms needed to enable trap mutation decreases with increasing temperature. For instance, at $1500 \mathrm{~K}$ even $\mathrm{He}_{4}$ clusters are able to emit a SIA to become a $\mathrm{He}_{4} \mathrm{~V}_{1}$, similar to results reported in Refs. [41,52]. This trend strongly promotes trap mutation and therefore, He trapping with increasing temperature. The emission of He atoms from $\mathrm{He}_{n} \mathrm{~V}_{m}$ clusters also increases with temperature, favoring cluster coalescence. The higher concentration of He atoms in large clusters at these temperatures (Figure 6) leads to efficient fuzz growth. A different regime in He retention is observed at 1700 and $1900 \mathrm{~K}$. Although only short simulations were carried out, the trend is clearly observed: a much higher He retention rate can be observed. The process described above also applies at these temperatures, but at a much more accelerated rate due to the higher temperatures. The processes governing the temperature dependence of fuzz growth are therefore, (i) trap mutation and (ii) He emission from $\mathrm{He}_{n} \mathrm{~V}_{m}$ clusters, with the subsequent coalescence and formation of larger $\mathrm{He}_{n} \mathrm{~V}_{m}$ clusters.

At 2100 and $2300 \mathrm{~K}$, large clusters are formed and thus fuzz still grows. However, the temperature is sufficiently high to dissociate the $\mathrm{He}_{n} \mathrm{~V}_{m}$ clusters by emitting He atoms. The reason behind such transition can be derived from Figure 7. The maximum emission of He atoms at 1900, 2100 and $2300 \mathrm{~K}$ occurs when the vacancy contains $5 \mathrm{He}$ atoms, as it has the lowest binding energy $(2.23 \mathrm{eV}$, see Table 1). At $1900 \mathrm{~K}$, the equilibrium between formation and dissociation of $\mathrm{He}$ atoms in $\mathrm{He}_{n} \mathrm{~V}_{m}$ clusters takes place in vacancies with 7 to $9 \mathrm{He}$ atoms $\left(\mathrm{He}_{7} \mathrm{~V}_{1}\right.$ to $\left.\mathrm{He}_{9} \mathrm{~V}_{1}\right)$, which leads to the formation of large and stable clusters and thus, fuzz growth and He retention. At $2100 \mathrm{~K}$ starts the change in regime. The increase in temperature causes the equilibrium to appear at vacancies with $5 \mathrm{He}$ atoms $\left(\mathrm{He}_{5} \mathrm{~V}_{1}\right)$, which leads to the formation of large but instable clusters: fuzz grows but the clusters are dissolved at a higher rate and $\mathrm{He}$ retention decreases. At $2300 \mathrm{~K}$, the change in regime continues: the equilibrium appears at vacancies with 2 to $4 \mathrm{He}$ atoms $\left(\mathrm{He}_{2} \mathrm{~V}_{1}\right.$ to $\left.\mathrm{He}_{4} \mathrm{~V}_{1}\right)$ which have binding energies of the order of $3 \mathrm{eV}$ (see Table 1). This leads to a lower number of large $\mathrm{He}_{n} \mathrm{~V}_{m}$ clusters, i.e., a lower fuzz growth rate and a lower He retention. Bursting of some of these scares but large $\mathrm{He}_{n} \mathrm{~V}_{m}$ clusters may be behind the formation of pitholes observed experimentally at high temperatures [17].

Finally, at a high temperature $(2500 \mathrm{~K})$ the transition to the new regime ends. He emission from the formed $\mathrm{He}_{n} \mathrm{~V}_{m}$ clusters dominates, suppressing further cluster formation or growth, and thus, fuzz formation. In fact, almost no He is retained at $2500 \mathrm{~K}$ (Figure 6). In this case, the emitted He atoms 
cannot be trapped, but they migrate until they have reached the surface and desorb or migrate to the bulk.

In Figure 8, the vacancy concentration profile in the fuzz region at 900, 1120, 1320 and $1500 \mathrm{~K}$ is shown. The profile peaks become less pronounced with increasing temperature. The highest vacancy concentration is reached at $900 \mathrm{~K}$ with $\sim 6.8 \mathrm{~nm}^{-3}$, i.e., $10.8 \%$ of the $\mathrm{W}$ atoms are displaced from their lattice positions forming vacancies. The vacancy concentration reaches successive peaks, which tend to be lower with the increasing fuzz height and temperature. This result indicates that, as fuzz height increases, the incoming He ions interact mainly with the $\mathrm{He}_{n} \mathrm{~V}_{m}$ clusters closest to the surface. For a better comparison of vacancy concentration at different temperatures, we have also calculated the mean vacancy concentration in the fuzz region (inset of Figure 8). As a result, we observe that the higher the temperature, the lower the mean vacancy concentration.

\section{Conclusions}

In summary, we report an OKMC study on the effect of temperature on fuzz growth in low energy Heirradiated W. In our simulations, fuzz growth is observed in the temperature range from 900 to $2300 \mathrm{~K}$, in agreement with experimental observations. We have identified that fuzz growth is driven by the formation of large $\mathrm{He}_{n} \mathrm{~V}_{m}$ clusters, which are only stable at intermediate temperatures (900-1900 K). Two main mechanisms lead to this temperature dependence: (i) emission of SIAs ("trap mutation") and (ii) coalescence of small $\mathrm{He}_{n} \mathrm{~V}_{m}$ clusters due to emission of He atoms and formation of new $\mathrm{He}_{n} \mathrm{~V}_{m}$ clusters in their vicinity. At low temperatures $(700 \mathrm{~K})$, fuzz growth is prevented by the stability of small $\mathrm{He}_{9} \mathrm{~V}_{1}$ clusters, leading to no He emission. In contrast, at high temperatures $(2500 \mathrm{~K})$ fuzz does not grow due to the high He emission from $\mathrm{He}_{n} \mathrm{~V}_{m}$ clusters, leading to their dissolution and thus, preventing He retention. We can conclude that fuzz growth is not only influenced by He retention, but by the size and stability of $\mathrm{He}_{n} \mathrm{~V}_{m}$ clusters in which He atoms are retained. Regarding the temporal evolution of fuzz growth, our simulations predict an incubation fluence consistent with recent experimental observations. Note that the simulations have been carried out in a pseudo-3D approach. Although the dynamics of $\mathrm{He}_{n} \mathrm{~V}_{m}$ clusters formation and dissociation are not influenced by the box size, incubation fluence and fuzz growth rate could be probably better reproduced in full-3D simulations.

\section{Acknowledgements}

The authors acknowledge financial support by the Spanish MINECO project ENE-2012-39787-C0603. The authors also acknowledge CSC, Academy of Finland. This work has been carried out within the framework of the EUROfusion Consortium and has received funding from the Euratom research and training programme 2014-2018 under the grant agreement No 633053. The views and opinions expressed herein do not necessarily reflect those of the European Commission. 


\section{References}

[1] J.W. Davis, V.R. Barabash, A. Makhankov, L. Plöchl, K.T. Slattery, Assessment of tungsten for use in the ITER plasma facing components, J. Nucl. Mater. 258-263, Part 1 (1998) 308-312. doi:10.1016/S0022-3115(98)00285-2.

[2] H. Bolt, V. Barabash, W. Krauss, J. Linke, R. Neu, S. Suzuki, N. Yoshida, A.U. Team, Materials for the plasma-facing components of fusion reactors, J. Nucl. Mater. 329-333, Part A (2004) 6673. doi:10.1016/j.jnucmat.2004.04.005.

[3] J. Roth, E. Tsitrone, T. Loarer, V. Philipps, S. Brezinsek, Alberto Loarte, G.F. Counsell, R.P. Doerner, K. Schmid, O.V. Ogorodnikova, R.A. Causey, Tritium inventory in ITER plasma-facing materials and tritium removal procedures, Plasma Phys. Control. Fusion. 50 (2008) 103001. doi:10.1088/0741-3335/50/10/103001.

[4] M.J. Baldwin, R.P. Doerner, Helium induced nanoscopic morphology on tungsten under fusion relevant plasma conditions, Nucl. Fusion. 48 (2008) 035001. doi:10.1088/0029-5515/48/3/035001.

[5] G.M. Wright, D. Brunner, M.J. Baldwin, R.P. Doerner, B. Labombard, B. Lipschultz, J.L. Terry, D.G. Whyte, Tungsten nano-tendril growth in the Alcator C-Mod divertor, Nucl. Fusion. 52 (2012) 042003. doi:10.1088/0029-5515/52/4/042003.

[6] G.D. Temmerman, K. Bystrov, J.J. Zielinski, M. Balden, G. Matern, C. Arnas, L. Marot, Nanostructuring of molybdenum and tungsten surfaces by low-energy helium ions, J. Vac. Sci. Technol. A. 30 (2012) 041306. doi:10.1116/1.4731196.

[7] P. Fiflis, M.P. Christenson, N. Connolly, D.N. Ruzic, Nanostructuring of Palladium with LowTemperature Helium Plasma, Nanomaterials. 5 (2015) 2007-2018. doi:10.3390/nano5042007.

[8] M.J. Baldwin, R.P. Doerner, D. Nishijima, K. Tokunaga, Y. Ueda, The effects of high fluence mixed-species (deuterium, helium, beryllium) plasma interactions with tungsten, J. Nucl. Mater. 390-391 (2009) 886-890. doi:10.1016/j.jnucmat.2009.01.247.

[9] T.J. Petty, M.J. Baldwin, M.I. Hasan, R.P. Doerner, J.W. Bradley, Tungsten “fuzz” growth reexamined: the dependence on ion fluence in non-erosive and erosive helium plasma, Nucl. Fusion. 55 (2015) 093033. doi:10.1088/0029-5515/55/9/093033.

[10] N. Ohno, S. Kajita, D. Nishijima, S. Takamura, Surface modification at tungsten and tungsten coated graphite due to low energy and high fluence plasma and laser pulse irradiation, J. Nucl. Mater. 363-365 (2007) 1153-1159. doi:10.1016/j.jnucmat.2007.01.148.

[11] S. Kajita, S. Takamura, N. Ohno, D. Nishijima, H. Iwakiri, N. Yoshida, Sub-ms laser pulse irradiation on tungsten target damaged by exposure to helium plasma, Nucl. Fusion. 47 (2007) 1358. doi:10.1088/0029-5515/47/9/038.

[12] W. Sakaguchi, S. Kajita, N. Ohno, M. Takagi, In situ reflectivity of tungsten mirrors under helium plasma exposure, J. Nucl. Mater. 390-391 (2009) 1149-1152. doi:10.1016/j.jnucmat.2009.01.276.

[13] S. Kajita, N. Ohno, W. Sakaguchi, M. Tagaki, Visualized Blow-off from Helium Irradiated Tungsten in Response to ELM-like Heat Load, Plasma Fusion Res. 4 (n.d.). https://www.jstage.jst.go.jp/article/pfr/4/0/4_0_004/_article (accessed September 14, 2016).

[14] T.J. Petty, J.W. Bradley, Tungsten nanostructure formation in a magnetron sputtering device, J. Nucl. Mater. 453 (2014) 320-322. doi:10.1016/j.jnucmat.2014.07.023.

[15] M.J. Baldwin, T.C. Lynch, R.P. Doerner, J.H. Yu, Nanostructure formation on tungsten exposed to low-pressure rf helium plasmas: A study of ion energy threshold and early stage growth, J. Nucl. Mater. 415 (2011) S104-S107. doi:10.1016/j.jnucmat.2010.10.050.

[16] M.J. Baldwin, R.P. Doerner, Formation of helium induced nanostructure "fuzz" on various tungsten grades, J. Nucl. Mater. 404 (2010) 165-173. doi:10.1016/j.jnucmat.2010.06.034. 
[17] S. Kajita, W. Sakaguchi, N. Ohno, N. Yoshida, T. Saeki, Formation process of tungsten nanostructure by the exposure to helium plasma under fusion relevant plasma conditions, Nucl. Fusion. 49 (2009) 095005. doi:10.1088/0029-5515/49/9/095005.

[18] Wright, Comparison of tungsten nano-tendrils grown in Alcator C-Mod and linear plasma devices, J. Nucl. Mater. 438, Supplement (2013) S84-S89. doi:http://dx.doi.org/10.1016/j.jnucmat.2013.01.013.

[19] S. Kajita, N. Yoshida, R. Yoshihara, N. Ohno, M. Yamagiwa, TEM observation of the growth process of helium nanobubbles on tungsten: Nanostructure formation mechanism, J. Nucl. Mater. 418 (2011) 152-158. doi:10.1016/j.jnucmat.2011.06.026.

[20] A. Lasa, K.O.E. Henriksson, K. Nordlund, MD simulations of onset of tungsten fuzz formation under helium irradiation, Nucl. Instrum. Methods Phys. Res. Sect. B Beam Interact. Mater. At. 303 (2013) 156-161. doi:10.1016/j.nimb.2012.11.029.

[21] A. Lasa, S.K. Tähtinen, K. Nordlund, Loop punching and bubble rupture causing surface roughening — A model for W fuzz growth, EPL Europhys. Lett. 105 (2014) 25002. doi:10.1209/0295-5075/105/25002.

[22] A.M. Ito, A. Takayama, Y. Oda, T. Tamura, R. Kobayashi, T. Hattori, S. Ogata, N. Ohno, S. Kajita, M. Yajima, Y. Noiri, Y. Yoshimoto, S. Saito, S. Takamura, T. Murashima, M. Miyamoto, H. Nakamura, Molecular dynamics and Monte Carlo hybrid simulation for fuzzy tungsten nanostructure formation, Nucl. Fusion. 55 (2015) 073013. doi:10.1088/0029-5515/55/7/073013.

[23] A.M. Ito, A. Takayama, Y. Oda, T. Tamura, R. Kobayashi, T. Hattori, S. Ogata, N. Ohno, S. Kajita, M. Yajima, Y. Noiri, Y. Yoshimoto, S. Saito, S. Takamura, T. Murashima, M. Miyamoto, H. Nakamura, Hybrid simulation research on formation mechanism of tungsten nanostructure induced by helium plasma irradiation, J. Nucl. Mater. 463 (2015) 109-115. doi:10.1016/j.jnucmat.2015.01.018.

[24] S.I. Krasheninnikov, T. Faney, B.D. Wirth, On helium cluster dynamics in tungsten plasma facing components of fusion devices, Nucl. Fusion. 54 (2014) 073019. doi:10.1088/0029. $5515 / 54 / 7 / 073019$.

[25] K.D. Hammond, B.D. Wirth, Crystal orientation effects on helium ion depth distributions and adatom formation processes in plasma-facing tungsten, J. Appl. Phys. 116 (2014) 1-8. doi:10.1063/1.4897419.

[26] B.D. Wirth, K.D. Hammond, S.I. Krasheninnikov, D. Maroudas, Challenges and opportunities of modeling plasma-surface interactions in tungsten using high-performance computing, J. Nucl. Mater. 463 (2015) 30-38. doi:10.1016/j.jnucmat.2014.11.072.

[27] S.I. Krasheninnikov, R.D. Smirnov, He cluster dynamics in fusion related plasma facing materials, Nucl. Fusion. 55 (2015) 073005. doi:10.1088/0029-5515/55/7/073005.

[28] T. Faney, S.I. Krasheninnikov, B.D. Wirth, Spatially dependent cluster dynamics model of He plasma surface interaction in tungsten for fusion relevant conditions, Nucl. Fusion. 55 (2015) 013014. doi:10.1088/0029-5515/55/1/013014.

[29] D. Maroudas, S. Blondel, L. Hu, K.D. Hammond, B.D. Wirth, Helium segregation on surfaces of plasma-exposed tungsten, J. Phys. Condens. Matter. 28 (2016) 064004. doi:10.1088/09538984/28/6/064004.

[30] I. Martin-Bragado, A. Rivera, G. Valles, J.L. Gomez-Selles, M.J. Caturla, MMonCa: An Object Kinetic Monte Carlo simulator for damage irradiation evolution and defect diffusion, Comput. Phys. Commun. 184 (2013) 2703-2710. doi:10.1016/j.cpc.2013.07.011. 
[31] G. Valles, C. González, I. Martin-Bragado, R. Iglesias, J.M. Perlado, A. Rivera, The influence of high grain boundary density on helium retention in tungsten, J. Nucl. Mater. 457 (2015) 80-87. doi:10.1016/j.jnucmat.2014.10.038.

[32] C.S. Becquart, C. Domain, Migration Energy of He in W Revisited by Itextit $\{$ Ab Initio Calculations, Phys. Rev. Lett. 97 (2006) 196402. doi:10.1103/PhysRevLett.97.196402.

[33] C.S. Becquart, C. Domain, U. Sarkar, A. DeBacker, M. Hou, Microstructural evolution of irradiated tungsten: Ab initio parameterisation of an OKMC model, J. Nucl. Mater. 403 (2010) 7588. doi:10.1016/j.jnucmat.2010.06.003.

[34] C.S. Becquart, M.F. Barthe, A.D. Backer, Modelling radiation damage and He production in tungsten, Phys. Scr. 2011 (2011) 014048. doi:10.1088/0031-8949/2011/T145/014048.

[35] F. Sefta, K.D. Hammond, N. Juslin, B.D. Wirth, Tungsten surface evolution by helium bubble nucleation, growth and rupture, Nucl. Fusion. 53 (2013) 073015. doi:10.1088/00295515/53/7/073015.

[36] A.V. Barashev, H. Xu, R.E. Stoller, The behavior of small helium clusters near free surfaces in tungsten, J. Nucl. Mater. 454 (2014) 421-426. doi:10.1016/j.jnucmat.2014.08.033.

[37] J. Boisse, C. Domain, C.S. Becquart, Modelling self trapping and trap mutation in tungsten using DFT and Molecular Dynamics with an empirical potential based on DFT, J. Nucl. Mater. 455 (2014) 10-15. doi:10.1016/j.jnucmat.2014.02.031.

[38] D. Perez, T. Vogel, B.P. Uberuaga, Diffusion and transformation kinetics of small helium clusters in bulk tungsten, Phys. Rev. B. 90 (2014) 014102. doi:10.1103/PhysRevB.90.014102.

[39] K. Nordlund, J. Keinonen, M. Ghaly, R.S. Averback, Coherent displacement of atoms during ion irradiation, Nature. 398 (1999) 49-51. doi:10.1038/17983.

[40] L. Hu, K.D. Hammond, B.D. Wirth, D. Maroudas, Dynamics of small mobile helium clusters near tungsten surfaces, Surf. Sci. 626 (2014) L21-L25. doi:10.1016/j.susc.2014.03.020.

[41] L. Hu, K.D. Hammond, B.D. Wirth, D. Maroudas, Molecular-dynamics analysis of mobile helium cluster reactions near surfaces of plasma-exposed tungsten, J. Appl. Phys. 118 (2015) 163301. doi:10.1063/1.4933393.

[42] A. Takayama, A.M. Ito, S. Saito, N. Ohno, H. Nakamura, First-Principles Investigation on Trapping of Multiple Helium Atoms within a Tungsten Monovacancy, Jpn. J. Appl. Phys. 52 (2013) 01AL03. doi:10.7567/JJAP.52.01AL03.

[43] T. Tamura, R. Kobayashi, S. Ogata, A.M. Ito, First-principles investigation of possible clustering of noble gas atoms implanted in bcc tungsten, Model. Simul. Mater. Sci. Eng. 22 (2014) 015002. doi:10.1088/0965-0393/22/1/015002.

[44] M.S. Abd El Keriem, D.P. van der Werf, F. Pleiter, Helium-vacancy interaction in tungsten, Phys. Rev. B. 47 (1993) 14771-14777. doi:10.1103/PhysRevB.47.14771.

[45] N. Juslin, B.D. Wirth, Interatomic potentials for simulation of He bubble formation in W, J. Nucl. Mater. 432 (2013) 61-66. doi:10.1016/j.jnucmat.2012.07.023.

[46] P. Fiflis, D. Curreli, D.N. Ruzic, Direct time-resolved observation of tungsten nanostructured growth due to helium plasma exposure, Nucl. Fusion. 55 (2015) 033020. doi:10.1088/0029. 5515/55/3/033020.

[47] J.F. Ziegler, M.D. Ziegler, J.P. Biersack, SRIM - The stopping and range of ions in matter (2010), Nucl. Instrum. Methods Phys. Res. Sect. B Beam Interact. Mater. At. 268 (2010) 1818-1823. doi:10.1016/j.nimb.2010.02.091.

[48] G. Valles, A. L. Cazalilla, C. Gonzalez, I. Martin-Bragado, A. Prada, R. Iglesias, J.M. Perlado, A. Rivera, A multiscale approach to defect evolution in tungsten under helium irradiation, Nucl. 
Instrum. Methods Phys. Res. Sect. B Beam Interact. Mater. At. 352 (2015) 100-103. doi:10.1016/j.nimb.2014.12.034.

[49] A. Rivera, G. Valles, M.J. Caturla, I. Martin-Bragado, Effect of ion flux on helium retention in helium-irradiated tungsten, Nucl. Instrum. Methods Phys. Res. Sect. B Beam Interact. Mater. At. 303 (2013) 81-83. doi:10.1016/j.nimb.2012.10.038.

[50] M. Thompson, P. Kluth, R.P. Doerner, N. Kirby, D. Riley, C.S. Corr, Measuring helium bubble diameter distributions in tungsten with grazing incidence small angle x-ray scattering (GISAXS), Phys. Scr. T. 2016 (2016) 014014 (4 pp.). doi:10.1088/0031-8949/2016/T167/014014.

[51] M. Thompson, R. Sakamoto, E. Bernard, N. Kirby, P. Kluth, D. Riley, C. Corr, GISAXS modelling of helium-induced nano-bubble formation in tungsten and comparison with TEM, J. Nucl. Mater. 473 (2016) 6-12. doi:10.1016/j.jnucmat.2016.01.038.

[52] W.D. Wilson, C.L. Bisson, M.I. Baskes, Self-trapping of helium in metals, Phys. Rev. B. 24 (1981) 5616-5624. doi:10.1103/PhysRevB.24.5616. 


\section{TALBES}

Table 1. Binding energy of a He atoms to a vacancy $\left(\mathrm{He}_{n} \mathrm{~V}_{1}\right)$ as a function of the number of $\mathrm{He}$ atoms in the vacancy.

\begin{tabular}{ll}
\hline Cluster & Binding energy $(\mathrm{eV})$ \\
\hline $\mathrm{HeV}(\mathrm{He} \rightarrow \mathrm{V})$ & 4.67 \\
$\mathrm{He}_{2} \mathrm{~V}(\mathrm{He} \rightarrow \mathrm{HeV})$ & 3.22 \\
$\mathrm{He}_{3} \mathrm{~V}\left(\mathrm{He} \rightarrow \mathrm{He}_{2} \mathrm{~V}\right)$ & 3.17 \\
$\mathrm{He}_{4} \mathrm{~V}\left(\mathrm{He} \rightarrow \mathrm{He}_{3} \mathrm{~V}\right)$ & 3.23 \\
$\mathrm{He}_{5} \mathrm{~V}\left(\mathrm{He} \rightarrow \mathrm{He}_{4} \mathrm{~V}\right)$ & 2.23 \\
$\mathrm{He}_{6} \mathrm{~V}\left(\mathrm{He} \rightarrow \mathrm{He}_{5} \mathrm{~V}\right)$ & 2.77 \\
$\mathrm{He}_{7} \mathrm{~V}\left(\mathrm{He} \rightarrow \mathrm{He}_{6} \mathrm{~V}\right)$ & 2.35 \\
$\mathrm{He}_{8} \mathrm{~V}\left(\mathrm{He} \rightarrow \mathrm{He}_{7} \mathrm{~V}\right)$ & 2.53 \\
$\mathrm{He}_{9} \mathrm{~V}\left(\mathrm{He} \rightarrow \mathrm{He}_{8} \mathrm{~V}\right)$ & 2.13 \\
\hline
\end{tabular}




\section{FIGURES}
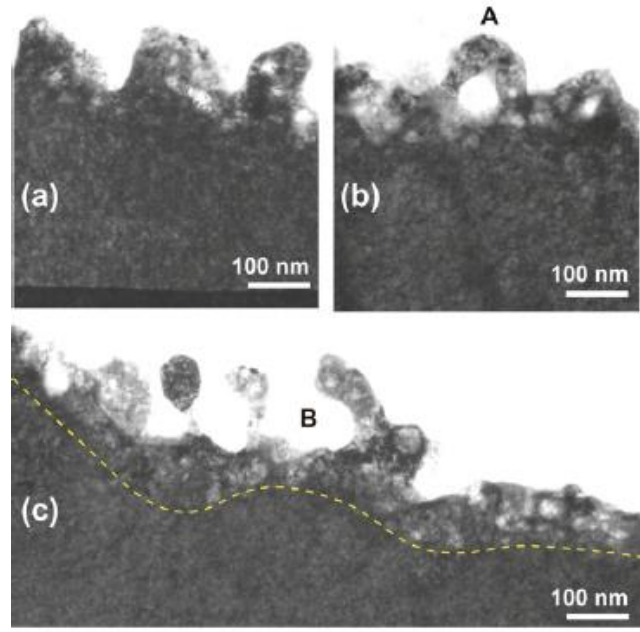

Figure 1. Cross sectional TEM micrographs of the helium irradiated tungsten sample at different positions under a helium fluence $6 \times 10^{24} \mathrm{~m}^{-2}$ [19]. Reprinted from Journal of Nuclear Materials, 418, S. Kajita, N. Yoshida, R. Yoshihara, N. Ohno, M- Yamagiwa, TEM observation of the growth process of helium nanobubbles on tungsten: Nanostructure formation mechanism, 152-158, Copyright (2011), with permission from Elsevier. 

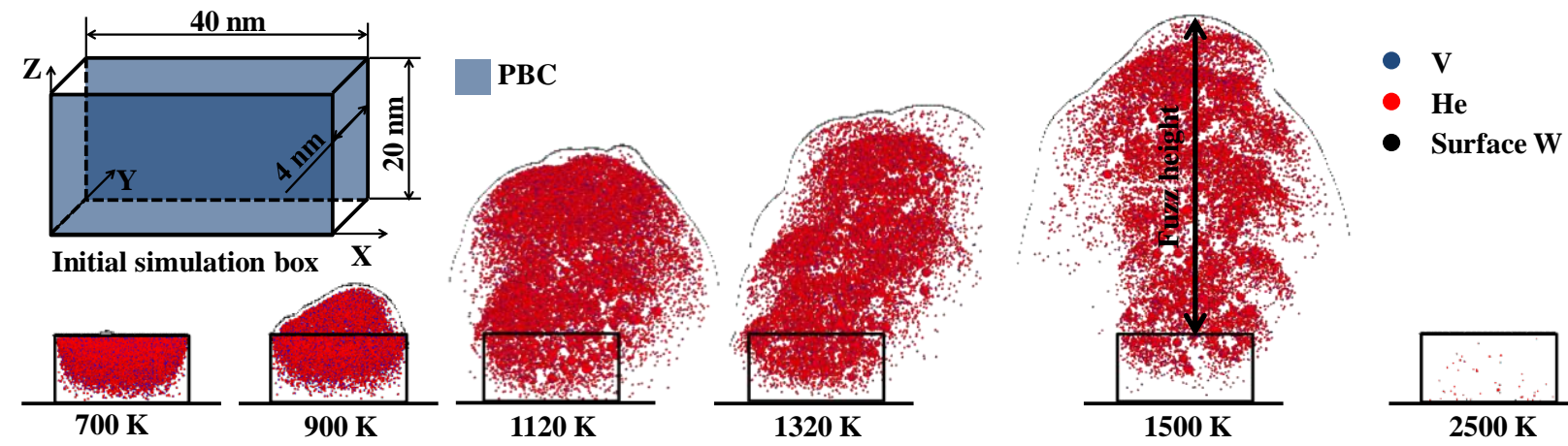

- Surface W

Figure 2. Dimensions of the simulation boxes and schematic representation of $\mathrm{W}$ surface growth (fuzz growth) at different temperatures after $3 \mathrm{~s}$ of $\mathrm{He}(60 \mathrm{eV})$ irradiation. 
Helium

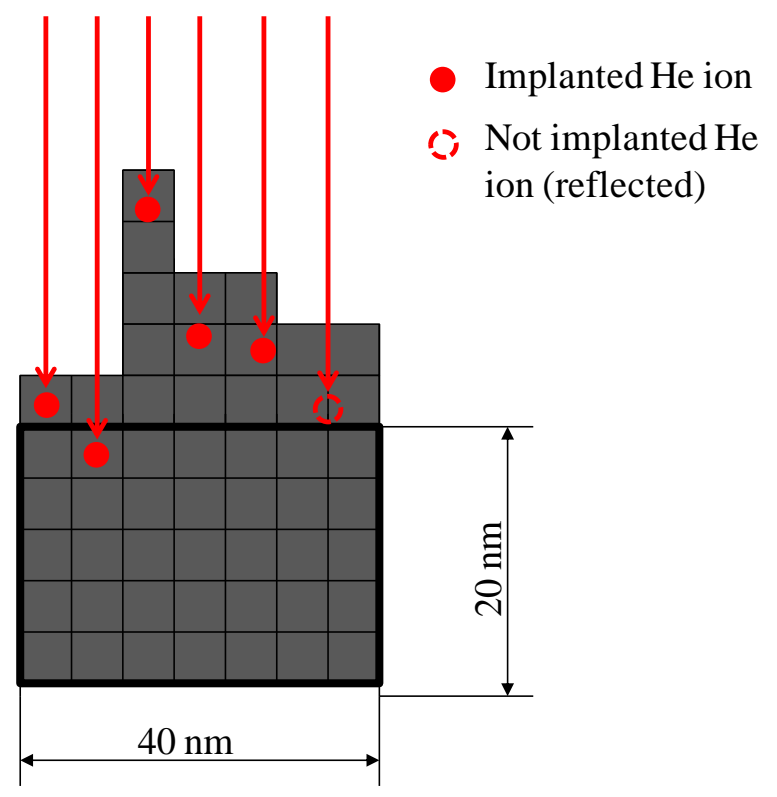

Figure 3. MMonCa takes into account the height of the $\mathrm{W}$ surface and the reflection yield when implanting He ions at depths calculated by SRIM. 

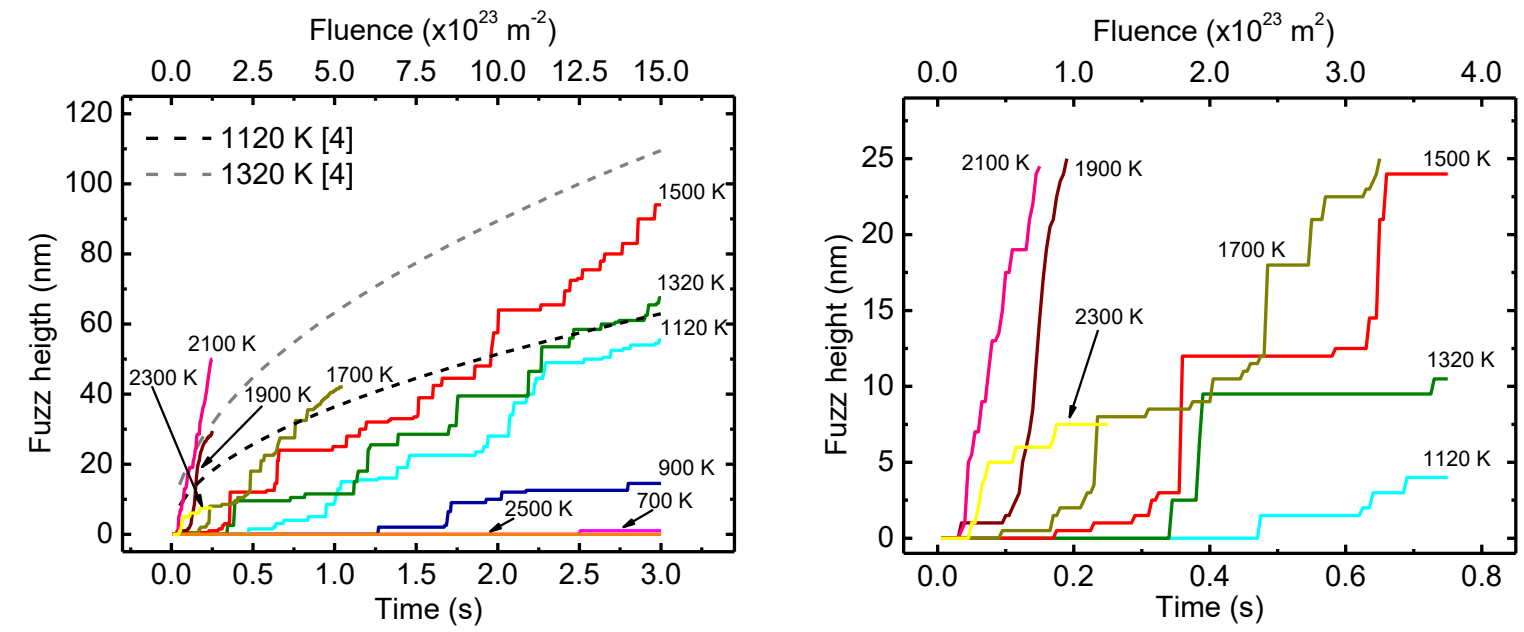

Figure 4. (a) Fuzz height as a function of time (i.e. fluence) at the simulated temperatures. Fuzz height obtained from experimental data at both 1120 and $1320 \mathrm{~K}$ [4], are also plotted. (b) A zoom in into the fuzz height evolution in the first $0.8 \mathrm{~s}$ of irradiation. 


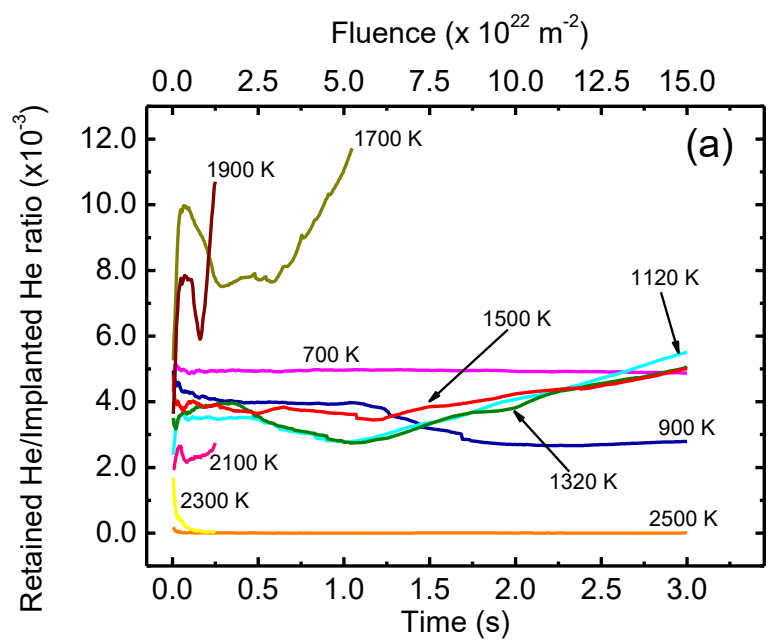

Figure 5. Retained He fraction as a function of time (and fluence) for different temperatures. 
Authors final preprint of paper published as JOURNAL OF NUCLEAR MATERIALS. 490 (2017) P. 108-114

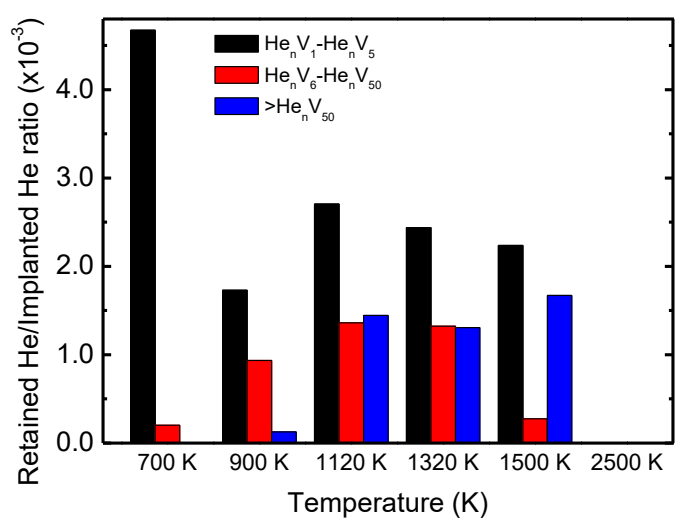

Figure 6. He retention at 700, 900,1120,1320, 1500 and $2500 \mathrm{~K}$ sorted by cluster size after up to $3 \mathrm{~s}$ of He irradiation. 


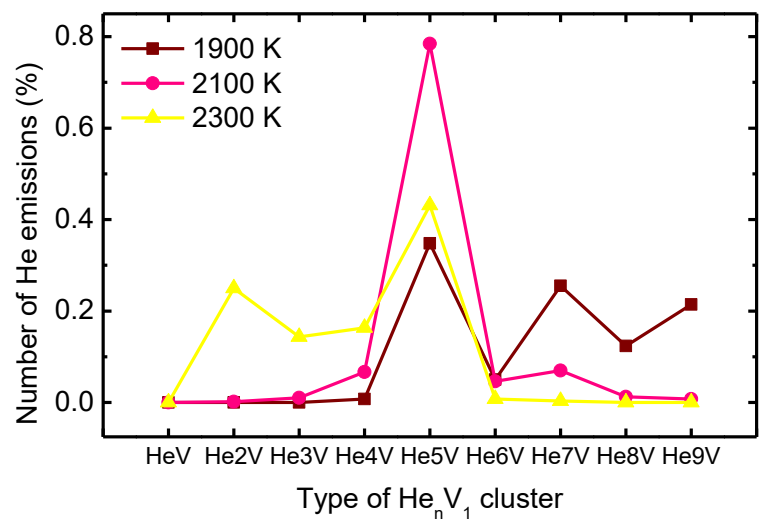

Figure 7. Number of $\mathrm{He}$ emissions for different $\mathrm{He}_{n} \mathrm{~V}_{1}$ clusters with respect with the total $\mathrm{He}$ emissions in $\mathrm{He}_{n} \mathrm{~V}_{1}$ clusters. 


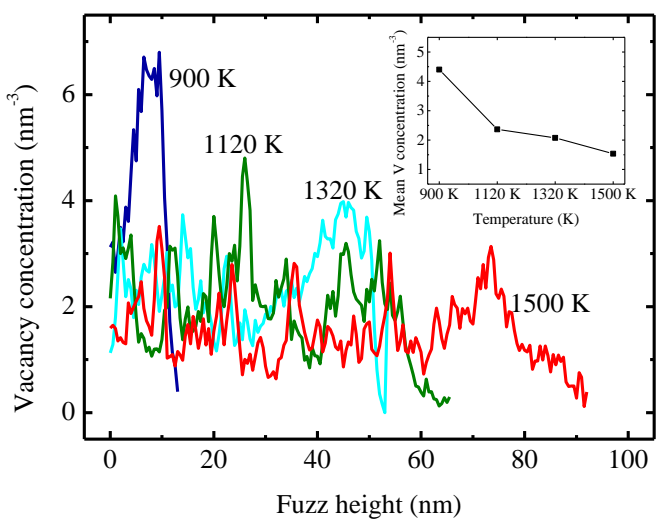

Figure 8. Vacancy concentration profile in the fuzz region as a function of fuzz height after He irradiation during $3 \mathrm{~s}$ at intermediate temperatures $(900,1120,1320$ and $1500 \mathrm{~K})$, at which fuzz growth takes place. Inset: mean vacancy concentration (total number of vacancies/total W-fuzz volume) for 900, 1120, 1320 and $1500 \mathrm{~K}$. 